\title{
Suivi toxicocinétique de la clozapine : étude d'une tentative de suicide
}

\section{Toxicokinetics of clozapine : a case report of a non fatal suicidal intoxication}

Laurence LABAT ${ }^{*(1,2)}$, Marc DEVEAUX ${ }^{(3)}$, Régis BEDRY $^{(4)}$, Nicholas MOORE $^{(2)}$

(1) Laboratoire de Chimie Analytique, UFR de Pharmacie, 3, Place de la Victoire - 33076 BORDEAUX Cedex

(2) Laboratoire de Toxicologie, Hôpital Pellegrin, Place Amélie Raba Léon - 33076 BORDEAUX Cedex

(3) Institut de Médecine Légale, Place Théo Varlet - 59000 LILLE

(4) Centre Antipoison, Hôpital Pellegrin, Place Amélie Raba Léon - 33076 BORDEAUX Cedex

*Auteur à qui adresser la correspondance : Laurence LABAT, Laboratoire de Chimie Analytique, UFR de Sciences Pharmaceutiques, 3, Place de la Victoire - 33076 BORDEAUX Cedex Tél : 0557571818 - Fax : 0556947090 - e-mail : laurence.labat@chimana.u-bordeaux2.fr

\section{$\boldsymbol{R E} \boldsymbol{E} U M \boldsymbol{E}^{\prime}$}

Un homme de 29 ans fait une tentative de suicide par ingestion supposée de $3 \mathrm{~g}$ de clozapine, de zopiclone, d'alprazolam et d'alcool. Il est admis à l'hôpital dans un coma profond hypotonique, en état de dépression respiratoire et de collapsus respiratoire et vasculaire. Après un traitement symptomatique, le patient quitte le service de réanimation 72 $h$ après l'ingestion des médicaments, sans séquelles. Des dosages de la clozapine plasmatique par chromatographie liquide haute performance (CLHP/UV) ont été réalisés sur 16 prélèvements successifs pendant 120 heures. Les résultats toxicocinétiques montrent une phase d'absorption de $4 \mathrm{~h}$ avec une concentration plasmatique maximum de 5200 $\mathrm{ng} / \mathrm{ml}$. Nous avons mis en évidence trois demi-vies d'élimination successives de la clozapine, estimées à 38, 24 et $13 \mathrm{~h}$.

\section{MOTS-CLÉS}

Clozapine, plasma, intoxication, CLHP/UV.

\section{SUMMARY}

A 29-year-old male attempted suicide with about $3 \mathrm{~g}$ clozapine, zopiclone, alprazolam and alcohol. He was admitted in a deep hypotonic coma with respiratory depression, inhalation pneumonia and vascular collapse. After a symptomatic treatment, the patient was discharged from the intensive care unit, with no sequelae. A high-pressure liquid chromatography method (HPLC/UV) was performed in order to quantify clozapine in the plasma during 120 hours on 16 blood samples. Toxicokinetic results showed a 4 hours absorption phase and a maximum blood concentration of $5200 \mathrm{ng} / \mathrm{ml}$. We demonstrate that clozapine has three successive elimination half-lives values of 38, 24 and 13 hours..

\section{KEY-WORDS}

Clozapine, blood, intoxication, HPLC/UV. 


\section{Introduction}

La clozapine, 8-chloro-11-(4-méthyl-1-piprazinyl)$5 H$-dibenzo[b,e]diazépine, est un neuroleptique du groupe des dibenzodiazépines. C'est un médicament antipsychotique atypique, actif sur les symptômes positifs et négatifs, primaires comme secondaires, de la schizophrénie (1). Elle présente une activité bloquante sur les récepteurs dopaminergiques et en particulier sur les récepteurs D4, ainsi que sur les récepteurs sérotoninergiques, cholinergiques et adrénergiques (2). Son action antipsychotique s'accompagne d'une action sédative rapide et intense. Cette molécule se différencie des autres classes de neuroleptiques par la rareté de ses effets secondaires extrapyramidaux $(3,4)$.

La clozapine est commercialisée en France sous le nom de Leponex ${ }^{\circledR}$ (Novartis Pharma, RueilMalmaison, France) en boites de 30 comprimés sécables à $25 \mathrm{mg}$ et à $100 \mathrm{mg}$. Elle est inscrite sur la liste I des substances vénéneuses et réservée à l'usage hospitalier, aux spécialistes et/ou aux services spécialisés en psychiatrie. Ses indications thérapeutiques sont les schizophrénies chroniques sévères en cas de résistance ou d'intolérance majeure aux neuroleptiques classiques (5). La posologie initiale est de 12,5 mg par jour pour atteindre progressivement 300 à 450 $\mathrm{mg}$ /jour $(5,6)$. La posologie maximale recommandée est de $600 \mathrm{mg} /$ jour. La clozapine peut provoquer une neutropénie pouvant évoluer vers une agranulocytose et c'est pourquoi un ensemble de mesures de sécurité doit obligatoirement être pris par le prescripteur $(4,5$, 7). L'utilisation de la clozapine implique que le patient ait une numération-formule leucocytaire normale ( leucocytes $\geq 3500 / \mathrm{mm}^{3}$ et neutrophiles $\geq$ $2000 / \mathrm{mm}^{3}$ ) et que l'on puisse réaliser une numérationformule leucocytaire régulièrement (toutes les semaines pendant 18 semaines, puis au moins une fois par mois pendant toute la durée du traitement). Pour cela, un carnet de surveillance hématologique est rempli et signé par le psychiatre qui doit vérifier la normalité de la numération-formule leucocytaire avant toute nouvelle prescription. La durée de prescription ne doit pas être supérieure à l'intervalle prévu entre deux examens hématologiques. Le pharmacien hospitalier doit contresigner le carnet de surveillance au moment de la délivrance du Leponex® ${ }^{\circledR}$.

Bien que connue depuis une trentaine d'années, peu de données toxicocinétiques cinétiques ont été décrites lors d'intoxications aiguës par la clozapine
(8-13). Nous rapportons ici l'observation d'un cas d'intoxication volontaire aiguë non mortelle, pour laquelle la toxicocinétique de la clozapine a été suivie par une méthode rapide de chromatographie liquide haute pression/détection par UV (CLHP/UV).

\section{Observation}

Un homme de 29 ans est retrouvé sur la voie publique dans un coma profond hypotonique (score de Glasgow $=3$ ) et en collapsus à $80 / 40 \mathrm{~mm} \mathrm{Hg}$, une à deux heures après l'ingestion supposée de $3 \mathrm{~g}$ de clozapine (Leponex ${ }^{\circledR}$ ), de zopiclone (Imovane ${ }^{\circledR}$ ), d'alprazolam (Xanax $\left.{ }^{\circledR}\right)$ et d'alcool. A son admission, l'ECG montre une tachycardie sinusale avec allongement de l'espace QT à 0,32 secondes. La radiographie montre une pneumopathie d'inhalation au niveau du lobe moyen gauche. Le bilan biologique initial montre une hyperglycémie $(2,68 \mathrm{~g} / \mathrm{l})$, une hypokaliémie $(3,2 \mathrm{mmol} / \mathrm{l})$ et une polynucléose à 16900 leucocytes $/ \mathrm{mm}^{3}$. Un premier bilan toxicologique par immunoanalyse (EMIT®, Dade Behring) ne montre que la présence de benzodiazépines dans le plasma. Il n'a pas été retrouvé d'antidépresseurs tricycliques dans le plasma, ni d'opiacés, ni de cannabinoïdes dans les urines (EMITß, Dade Behring). Il n'y avait pas d'alcool éthylique dans le plasma (REA®, Abbott). La recherche et le dosage des benzodiazépines dans le plasma par CLHP/UV(10) n'a permis de mettre en évidence que $4,3 \mu \mathrm{g} / \mathrm{ml}$ de nordiazépam. L'alprazolam et la zopiclone, recherchés en même temps que les benzodiazépines, n'ont pas été retrouvés.

Le traitement initial a consisté en une ventilation artificielle, un lavage gastrique de 15 litres et l'administration de $25 \mathrm{~g}$ de charbon activé (Carbomix ${ }^{\circledR}$ ) toutes les 4 heures pendant 24 heures : l'évolution des paramètres biologiques est satisfaisante, avec une normalisation en 5 heures de la glycémie et de la kaliémie. La ventilation artificielle a été maintenue pendant 24 heures. Un syndrome confusionnel a persisté encore 24 heures. Le patient a quitté le service de réanimation environ 72 heures après l'intoxication, pour être accueilli dans un service de psychiatrie.

Compte tenu du contexte clinique, la clozapine a été dosée par CLHP/UV sur seize prélèvements sanguins successifs, de la première heure d'hospitalisation à la cent vingtième heure. Le dosage de la clozapine n'a pas pu être réalisé dans le liquide de lavage gastrique. 


\section{Matériels et méthodes}

\section{Réactifs.}

Tous les réactifs sont de qualité analytique. Les solvants sont de qualité CLHP. La clozapine et la clothiapine (étalon interne) ont été gracieusement fournies par les laboratoires Sandoz. Les solutions-mères à $1 \mathrm{mg} / \mathrm{ml}$ sont préparées dans du méthanol et conservées à $-20^{\circ} \mathrm{C}$. Les solutions de travail sont préparées extemporanément dans du plasma (CTS de Bordeaux).

\section{Préparation de l'échantillon.}

Un ml de plasma, additionné de $1 \mu \mathrm{g}$ de clothiapine (étalon interne), est extrait en milieu basique $(\mathrm{NaOH}$ $1 \mathrm{~N}, 200 \mu \mathrm{l})$ par $5 \mathrm{ml}$ du mélange hexane : isopropanol (99: 1, v/v). La phase organique est reextraite par $100 \mu \mathrm{l}$ d'acide chlorhydrique $0,1 \mathrm{~N}$. Après élimination de la phase organique, $50 \mu 1$ de la phase acide sont injectés dans le système chromatographique.

\section{Appareillage.}

Les analyses sont réalisées sur un système chromatographique constitué d'un injecteur automatique WISP 710B (Waters, Milford, USA), d'une pompe ICS Model 2250 (ICS, Toulouse, France), d'une colonne Spherisorb C8 (150 mm x 4,6 mm) (Waters, St Quentin Yvelynes, France) maintenue à une température de $60^{\circ} \mathrm{C}$. La détection se fait à $220 \mathrm{~nm}$ avec un détecteur UV Spectra System UV 2000 (ThermoQuest, Les Ulys, France). Les chromatogrammes sont enregistrés et intégrés sur un intégrateur Data Jet Integrator (ThermoQuest, Les Ulys, France). La phase mobile est constituée par un mélange de tampon phosphate $0,066 \mathrm{M}$ et d'acétonitrile (55:45, v / v) contenant $0,05 \%(\mathrm{v} / \mathrm{v})$ de diéthylamine. Elle est ajustée à $\mathrm{pH} 6,4$ par de l'acide phosphorique. Le débit est de $2 \mathrm{ml} / \mathrm{min}$.

\section{Résultats}

\section{Validation de la méthode}

\section{Spécificité}

Dans les conditions opératoires précitées les temps de rétention de la clozapine et de son étalon interne sont respectivement de 4,01 min et 9,19 min. Le tableau I donne les temps de rétention des antidépresseurs extraits par la même méthode et dosés en routine au laboratoire. On remarque la possibilité d'interférences analytiques avec la doxépine, la paroxétine, la normaprotiline, la médifloxacine et la tianeptine.
Tableau I : Temps de rétention (tr) et temps de rétention relatifs (trr) de différents psychotropes pouvant être dosés par la même méthode.

\begin{tabular}{|lcc|}
\hline \multicolumn{1}{|c|}{ Molécules } & tr (min) & $\begin{array}{c}\text { trr } \\
\text { (références : tr clozapine) }\end{array}$ \\
\hline viloxazine & 1,31 & 0,326 \\
méthyl-amineptine & 1,48 & 0,369 \\
tianeptine métabolite & 2,03 & 0,506 \\
métapramide & 2,67 & 0,660 \\
clomipramine & 3,14 & 0,780 \\
opipramol & 3,23 & 0,805 \\
amoxapine & 3,49 & 0,870 \\
démexityline & 3,58 & 0,892 \\
tianeptine & 3,85 & 0,960 \\
doxépine & 3,97 & 0,990 \\
clozapine & 4,01 & 1 \\
médifloxacine & 4,10 & 0,978 \\
normaprotiline & 4,21 & 1,050 \\
paroxétine & 4,21 & 1,050 \\
desipramine & 4,45 & 1,109 \\
oxaflozane & 4,66 & 1,160 \\
dosulépine & 5,09 & 1,269 \\
miansérine & 5,18 & 1,290 \\
nortriptyline & 5,34 & 1,330 \\
maprotiline & 5,39 & 1,344 \\
imipramine & 5,62 & 1,401 \\
amitryptiline & 6,57 & 1,638 \\
clotiapine (étalon interne) & 9,19 & 2,241 \\
\hline
\end{tabular}

\section{Linéarité}

La gamme d'étalonnage en 5 points $(0,0,75,1,1,5,2$ $\mu \mathrm{g} / \mathrm{ml}$ ) est réalisée à partir de plasma vierge supplémenté par de la solution mère de clozapine : la méthode est linéaire dans cette gamme.

\section{Limite de détection}

La limite de détection est définie à $20 \mathrm{ng} / \mathrm{ml}$ (signal/bruit de fond $=3$ ).

\section{Reproductibilité}

L'étude de la reproductibilité sur trois plasmas supplémentés $(0,35,0,7$ et $1 \mu \mathrm{g} / \mathrm{ml})$ donne des résultats satisfaisants avec des coefficients de variation de 3,1\%, $3,3 \%$ et $4,1 \%$.

\section{Rendement d'extraction}

Le rendement d'extraction est de $71+/-7 \%$, estimé sur 5 plasmas supplémentés de 0,1 à $1 \mu \mathrm{g} / \mathrm{ml}$ par comparaison avec des solutions méthanoliques injectées directement. 


\section{Paramètres toxicocinétiques}

La figure 1 représente les variations de la concentration plasmatique de la clozapine chez le patient depuis son admission à l'hôpital $(\mathrm{t}=0 \mathrm{~h})$ jusqu'au temps $\mathrm{t}=126 \mathrm{~h}$. La clozapine est très rapidement absorbée après l'ingestion, et une concentration à $5200 \mathrm{ng} / \mathrm{ml}$ est atteinte au temps $\mathrm{t}=5 \mathrm{~h}$. La demi-vie d'absorption est évaluée graphiquement à $4 \mathrm{~h}$. Trois demi-vies d'élimination peuvent être également déterminées graphiquement : entre la $9^{\mathrm{emc}}$ et la $29^{\mathrm{m} m \mathrm{~m}}$ heure, alors que le charbon actif a été administré, la valeur de la demi-vie d'élimination est estimée à $38 \mathrm{~h}$. Après l'arrêt du traitement, entre la $32^{\text {eme }}$ et la $49^{\text {ene }}$ heure, la seconde demi-vie d'élimination est de $24 \mathrm{~h}$. Enfin, après la $57^{\mathrm{eme}}$ heure, la troisième demi-vie d'élimination est de $13 \mathrm{~h}$. Cette troisième pente apparaît alors que les symptômes neurologiques ont disparus. A la $126^{\mathrm{im}}$ heure, le taux plasmatique de clozapine est de $30 \mathrm{ng} / \mathrm{ml}$.

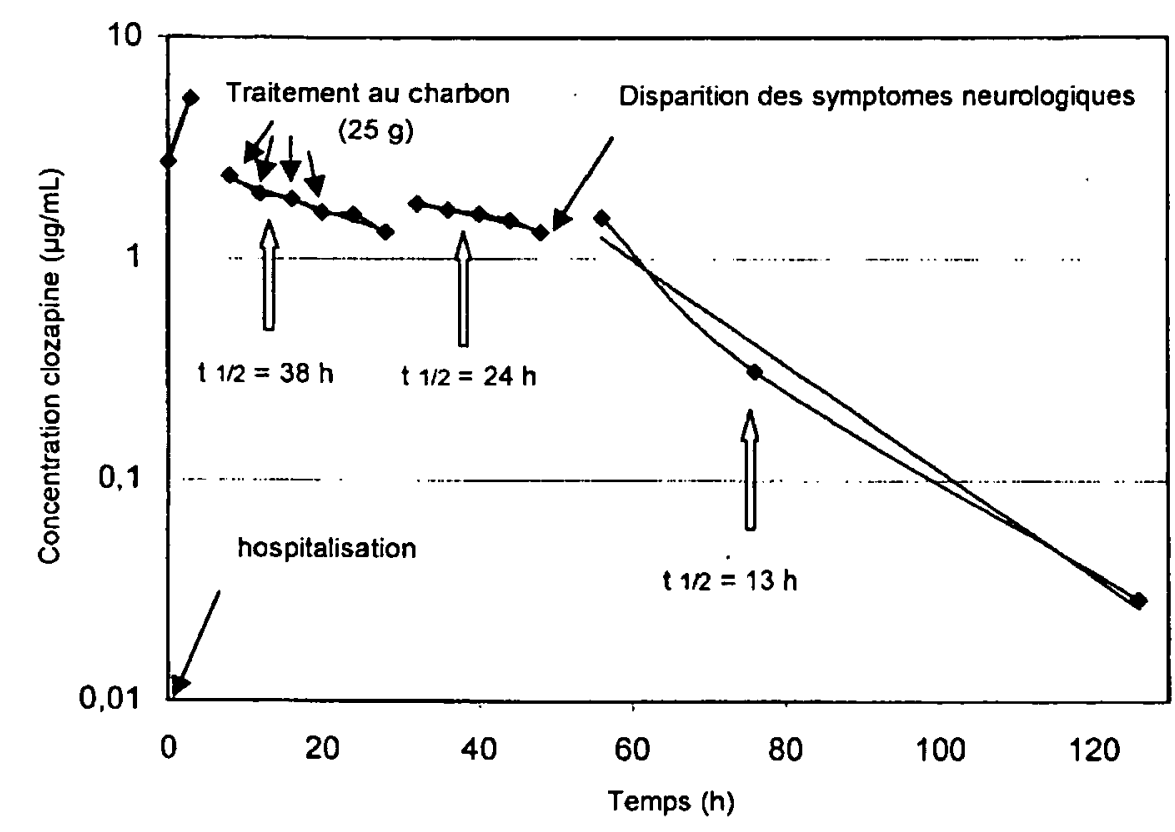

Figure 1 : Évolution des concentrations plasmatiques de la clozapine en fonction du temps.

\section{Discussion}

De nombreux cas d'intoxications aiguës et de décès par clozapine ont déjà été décrits pour des doses ingérées supérieures à $1 \mathrm{~g}$ (7-11,15-16). Les signes cliniques les plus classiques consistent, par ordre de fréquence décroissante en : coma, léthargie, tachycardie, agitation, confusion, perturbations de l'ECG et pneumopathie d'inhalation. $(7,9,17)$. Notre observation est tout à fait comparable et ces signes cliniques ont confortés les réanimateurs dans leur diagnostic d'intoxication aiguë par la clozapine chez un schizophrène connu.

La méthode de dosage plasmatique mise au point présente l'avantage d'éviter l'interférence analytique entre la clozapine et le nordiazépam décrite dans la littérature (18) : le nordiazépam sort dans le front de solvant (avant $3 \mathrm{~min}$ ), le temps de rétention de la clozapine est à 4,01 min.

Le mode isocratique est celui qui est classiquement utilisé (19-23). L'utilisation d'une colonne $\mathrm{C} 8$, d'une phase mobile à $\mathrm{pH}$ faiblement acide et d'une température de colonne de l'ordre de $60^{\circ} \mathrm{C}$ permet le dosage de la clozapine dans les procédures de routine du laboratoire $(21,24)$. La limite de détection est compatible avec la surveillance thérapeutique.

La N-desméthylclozapine, principal métabolite de la clozapine, n'a pas été dosée, car d'une part son activité pharmacologique est très inférieure à celle de la clozapine (25), d'autre part sa concentration n'est pas un marqueur clinique du risque d'agranulocytose (26) et enfin sa concentration plasmatique est habituellement inférieure à $25 \%$ de la concentration plasmatique de la clozapine $(8,23)$. La $\mathrm{N}$-oxyde clozapine, métabolite inactif et de concentration toujours inférieure à celle de la clozapine (18) n'a pas non plus été dosée.

La concentration maximum de $5200 \mathrm{ng} / \mathrm{ml}$ a permis de confirmer a posteriori le diagnostic d'intoxication massive à la clozapine, dont la concentration thérapeutique habituelle va de 200 à $400 \mathrm{ng} / \mathrm{ml}$ (6). Ce taux est supérieur à ceux décrits dans le cas de prise massive $(8,10)$.

L'évolution des concentrations plasmatiques dans notre observation est proche de celles décrites dans la littérature, bien que les doses ingérées soient différentes (9). Le temps nécessaire pour atteindre le pic plasmatique (5 à 6 h) n'est pas modifié par les doses ingérées (22). Le tableau II résume les paramètres toxicocinétiques relevés dans la littérature. Notre estimation graphique de la durée de la phase d'absorption est en accord avec les résultats d'Ischii (8) qui rapporte le cas d'une tentative de suicide chez une femme de 23 ans, avec une dose inconnue de clozapine. Il estime la demi-vie d'élimination à 18,5 h. Cette différence peut s'expliquer par le très faible nombre de prélèvements plasmatiques $(1,5 \mathrm{~h}, 3 \mathrm{~h}$ et $32 \mathrm{~h}$ après ingestion) alors que dans notre observation, 17 prélèvements réalisés à des intervalles de temps en moyenne de 4 heures permettent de décrire trois pentes d'élimination.

La première demi-vie d'élimination de $38 \mathrm{~h}$ est plus longue que celle rapportée par Ischii (8). On peut avancer deux explications. Tout d'abord, il peut exister une augmentation transitoire de l'absorption de la clozapine pendant le lavage gastrique par un effet de mise en solution du toxique (27). Cependant aucune étude n'a encore prouvé l'intérêt clinique de donner du charbon activé avant de faire un lavage gastrique. Ensuite, cette 
Tableau II : Paramètres toxicocinétiques de la clozapine décrits dans la littérature.

\begin{tabular}{|c|c|c|c|c|}
\hline $\begin{array}{c}\text { Dose ingérée } \\
\text { (g) }\end{array}$ & $\begin{array}{c}\text { Concentration } \\
\text { maximale de } \\
\text { clozapine }(\mu \mathrm{g} / \mathrm{ml})\end{array}$ & $\begin{array}{c}t \mathrm{i} / 2 \\
\text { absorption } \\
\text { (h) }\end{array}$ & $\begin{array}{c}\text { élimination } \\
\text { (h) }\end{array}$ & Références \\
\hline 3 (supposée) & 5,2 & 4 & 38,24 et 13 & notre étude \\
\hline inconnue & $\begin{array}{c}3,62 \\
\text { (3 h après ingestion) }\end{array}$ & - & 18,5 & (8) \\
\hline 16 & - & 0,75 & 20,8 & (9) \\
\hline 5,75 & - & 0,82 & 7,6 & (9) \\
\hline 5 & - & 1,97 & 10,7 & (9) \\
\hline inconnue & 20 (estimée) & - & 8,11 & (11) \\
\hline
\end{tabular}

phase est contemporaine du traitement par le charbon activé et on peut supposer la persistance d'une absorption pendant cette première période en dépit du traitement d'épuration. L'administration de charbon activé joue en effet un rôle majeur dans la prévention de l'absorption du médicament, mais la dose administrée était ici certainement insuffisante pour l'inhiber totalement. La décroissance des taux plasmatiques de clozapine est plus lente que celle que l'on aurait pu attendre, par comparaison avec l'utilisation de charbon activé dans le cas d'intoxication à d'autres médicaments psychotropes (28).

La seconde phase d'élimination, située entre la $32^{\mathrm{rme}} \mathrm{h}$ et la $49^{\text {ene }} \mathrm{h}$, est caractérisée par une demi-vie de $24 \mathrm{~h}$, et une concentration plasmatique initiale $(1760 \mathrm{ng} / \mathrm{ml})$ supérieure à la dernière concentration $(1310 \mathrm{ng} / \mathrm{ml})$ de la phase précédente. Cette différence peut certainement s'expliquer par l'existence d'un relargage intestinal de la clozapine à partir du charbon activé.

La dernière phase d'élimination débute environ $57 \mathrm{~h}$ après l'ingestion et présente une demi-vie de $13 \mathrm{~h}$. Cette dernière demi-vie d'élimination est proche de celles déjà décrites (tableau II). Bien que portant sur 17 points, il ne s'agit que d'une estimation graphique des paramètres toxicocinétiques, ne permettant pas un parallélisme absolu avec les données de la littérature, notamment quant au nombre exact de demi-vies d'élimination.

\section{Conclusion}

Ce cas de tentative de suicide par la clozapine est une illustration supplémentaire des symptômes cliniques induits par une prise massive de ce neuroleptique atypique. Les symptômes neurologiques disparaissent dès que la concentration plasmatique de clozapine atteint environ trois fois les valeurs thérapeutiques. La méthode d'extraction utilisée ne nécessite pas de phase d'évaporation et le dosage dans le plasma mis au point en CLHP-UV est simple et rapide. Les dosages effectués sur 17 prélèvements sanguins échelonnés sur 126 heures nous ont permis d'effectuer un suivi toxicocinétique de la clozapine et de mettre en évidence 3 demivies successives d'élimination.

\section{Remerciements}

Les auteurs remercient $\mathrm{M}^{\mathrm{me}} \mathrm{E}$. Deridet ainsi que le personnel du laboratoire de Pharmacologie-Toxicologie de l'hôpital Pellegrin pour leur aide technique. 


\section{Références}

1. Lançon C., Farisse J., Llorca P.M. Clozapine et traitement des symptômes négatifs. Encéphale 1997 ; Sp IV : 7-11.

2. Meltzer H.Y. An overview of the mechanism of action of clozapine. J.'Clin. Psychiatry $1994 ; 55$ : 47-52.

3. Safferman A., Lieberman J., Kane J., Szymanski S., Kinon B. Update on clinical efficacy and side-effects of clozapine. Schizophr. Bull. $1991 ; 17$ : 247-62.

4. Dev V.J., Krupp P. Adverse event profile and safety of clozapine. Rev. Contemp. Pharmacother. $1995 ; 6$ : 197 208.

5. Leponex ${ }^{\circledR}$, autorisations de mise sur le marché $\mathrm{n}^{\circ} 557$ 186.5 et $n^{\circ} 557187.1,1991$.

6. Olesen O.V. Therapeutic drug monitoring of clozapine treatment. Therapeutic threshold value for serum clozapine concentrations. Clin. Pharmacokinet. 1998 ; 34 : 497-502.

7. Le Blaye I., Donatini B., Hall M., Krupp P. Acute overdosage with clozapine : a review of the available clinical experience. Pharmaceutical Medicine 1992 ; 6 : 169-78.

8. Ishii A., Mizoguchi K., Kageoka M., Seno H., Kumazawa S., Suzuki O. Nonfatal suicidal intoxication by clozapine. J. Toxicol. Clin. Toxicol. 1997 ; 35 : 195-7.

9. Reith D., Monteleone J.P.R., Whyte I., Ebelling W., Holford N.H.G., Carter G. Features and toxicokinetics of clozapine in overdose. Ther. Drug Monit. 1998 ; 20 : 92-7.

10. Meeker J.E., Herrmann P.W., Som C.W., Reynolds P.C. Clozapine tissue concentrations following an apparent suicidal overdose of Clozaril. J. Anal. Toxicol. 1992 ; $16: 54-6$.

11. Mahoney M.C.,.Connolly B.F., Smith C.M. A clozapine overdose with markedly elevated serum levels. J. Clin. Pharmacol. 1999 ; 39 : 97-100.

12. Ganssmann B., Skopp G., Aderjan R., Mattern R. Fatal poisoning with clozapine and perazine. A case report. Arch. Kriminol 1998 ; 201 (1-2) : 11-20.

13. Piccini G., Ceroni P., Marchesi C., Maggini C., Maestri G. Acute clozapine overdosage. Br. J. Psychiatry 1997 ; $170: 290$.

14. Benhamou-Batut F., Demotes-Mainard F., Labat L., Vinçon G. et Bannwarth B. Determination of flunitrazepam in plasma by liquid chromatography. J. Pharm. Biomed. Anal. 1994 ; 12 : 931-6.

15. Stevens I., Gaertner H.J. Plasma level measurement in a patient with clozapine intoxication. J. Clin. Psychopharmacol. $1996 ; 16: 86-7$.
16. Keller T., Miki A., Binda S. Fatal overdose of clozapine. Forensic Sci. Int. 1997 ; 86 : 119-25.

17. Sautter T., Meier P.J., Wyss P.A. Dose dependent toxicity of clozapine (Leponex ${ }^{\circledR}$ ) in acute overdosage. XVII International Congress of the European Association of Poisons Centres and Clinical Toxicologists, Marseille, 47 Juin 1996.

18. Weigmann H., Bierbrauer J., Hartter S., Hiemke C. Automated determination of clozapine and major metabolites in serum and urine. Ther. Drug Monit. 1997 ; 19 : 480-8.

19. Lovdahl M.L. Perry P.J., Miller D.D. The assay of clozapine and N-desmethylclozapine in human plasma by HPLC. Ther. Drug Monit. $1991 ; 13: 69-72$.

20. Guitton C., Kinowski J.M., Aznar R., Bressolle F. Determination of clozapine and its major metabolites in human plasma and red blood cells by HPLC with UV absorbance detection. J. Chromatogr. B Biomed. Sci. Appl. $1997 ; 690$ (1-2) : 211-22.

21. Edno L., Combourieu I., Cazenave M., Tignol J. Assay for quantitation of clozapine and its metabolite $\mathrm{N}$-desmethylclozapine in human plasma by HPLC with UV detection. J. Pharm. Biomed. Anal. $1997 ; 16: 311-8$.

22. Akerman K.K. Analysis of clozapine and norclozapine by HPLC. J. Chromatogr. B Biomed. Sci. Appl. 1997 ; $696(2): 253-9$.

23. Guitton C., Kinowski J.M., Abbar M., Chabrand P., Bressolle F. Clozapine and metabolite concentrations during treatment of patient with chronic schizophrenia. J. Clin. Pharmacol. 1999 ; 39 : 721-8.

24. Bedry R., Labat-Deschamps L., Péhourcq F., Moore N., Pillet O., Favarel-Garrigues J.C. Non-fatal clozapine (Leponex $\left.{ }^{\circledR}\right)$ intoxication with toxicokinetic evaluation. Vet. Hum. Toxicol. $1999 ; 41: 20-2$.

25. Jann M.W., Grimsley S.R., Gray E.C., Chang W. Pharmacokinetics and pharmacodynamics of clozapine. Clin. Pharmacokinet. $1993 ; 24: 161-76$.

26. Combs M.D., Perry P.J., Bever K.A. N-desmethylclozapine, an insensitive marker of clozapine-induced agranulocytosis and granulocytopenia. Pharmacotherapy. 1997 ; $17: 1300-4$.

27. Vale J.A. Gastric lavage. AACT/EAPCCT position statements. XVII International Congress of the European Association of Poisons Centres and Clinical Toxicologists, Marseille, 4-7 Juin 1996.

28. Cooney D.O. Effect of multiple doses of charcoal in medical applications. New York : Marcel Dekker, 1995 : 318-56. 